\title{
Preoperative Blood Inflammatory Markers for the Differentiation of Uterine Leiomyosarcoma from Leiomyoma
}

\author{
Dong Soo Suh (iD ${ }^{1,2, *}$ \\ Yong Jung Song ${ }^{1,2, *}$ \\ Hyun-Jin Roh ${ }^{3}$ \\ Sang Hun Lee ${ }^{3}$ \\ Dae Hoon Jeong ${ }^{4}$ \\ Tae Hwa Lee ${ }^{5}$ \\ Kyung Un Choi ${ }^{6}$ \\ Ki Hyung Kim (D) ${ }^{1,2}$
}

'Department of Obstetrics and Gynecology, Pusan National University School of Medicine, Busan, South Korea;

${ }^{2}$ Biomedical Research Institute, Pusan National University Hospital, Busan, South Korea; ${ }^{3}$ Department of Obstetrics and Gynecology, University of Ulsan

College of Medicine, Ulsan University Hospital, Ulsan, South Korea;

${ }^{4}$ Department of Obstetrics and Gynecology, Busan Paik Hospital, Inje University College of Medicine, Busan, South Korea; ${ }^{5}$ Department of Obstetrics and Gynecology, Kosin University Gospel Hospital, Kosin University College of Medicine, Busan, South Korea; ${ }^{6}$ Department of Pathology, Pusan National University School of Medicine, Busan, South Korea

*These authors contributed equally to this work
Purpose: Preoperative diagnosis of uterine leiomyosarcoma (LMS) is challenging because the disease can mimic benign leiomyoma (LM). The objective of the present study was to investigate the role of preoperative clinical characteristics and hematologic parameters to differentiate uterine LMS and LM.

Methods: Preoperative clinical and laboratory variables were reviewed retrospectively in patients with LMS or LM, and the significances of intergroup differences were assessed. Receiver operating characteristic (ROC) curves were used to determine optimal cut-off values for each variable. Logistic regression analysis was applied to identify variables predicting the presence of LMS.

Results: The preoperative clinical and laboratory variables of 336 patients with uterine tumor were analyzed. Seventy-nine patients had LMS and 257 had LM. A significant difference was observed between LMS and LM in terms of the median value of age at diagnosis, menopausal status, white blood cell (WBC) count, absolute neutrophil count (ANC), C-reactive protein (CRP), lactate dehydrogenase (LDH), and neutrophil-tolymphocyte ratio (NLR) (all $P<0.001$ ). Multivariate analyses showed that menopausal status (odds ratio $[\mathrm{OR}]=3.40, P=0.002)$, WBC count $(\mathrm{OR}=2.09, P=0.012)$, ANC $(\mathrm{OR}=$ $3.17, P<0.001)$, CRP $(\mathrm{OR}=21.74, P<0.001)$, LDH $(\mathrm{OR}=10.77, P<0.001)$, and NLR $(\mathrm{OR}=2.58, P=0.001)$ predicted the presence of LMS.

Conclusion: Our results suggest that in older or postmenopausal patients, high WBC count, ANC, CRP, LDH, and NLR could be useful biomarkers for the differentiation of LMS and LM, which indicate that serum markers might be useful, cost-effective, and broadly available diagnostic markers for uterine LMS.

Keywords: C-reactive protein, lactate dehydrogenase, leiomyoma, leiomyosarcoma, neutrophil-to-lymphocyte ratio

\section{Introduction}

Uterine leiomyoma (LM) is a common gynecologic benign disease that affects $40-60 \%$ of all reproductive women, whereas uterine sarcoma is not commonly encountered. Leiomyosarcoma (LMS) is the most common type of uterine sarcoma, accounts for 1$2 \%$ of all uterine malignancies, and has a dismal prognosis. ${ }^{1}$ Furthermore, the risk of LMS in women with presumed LM is low but not negligible. Several studies have reported the prevalence of unexpected sarcoma in women undergoing hysterectomy for presumed benign LM ranges up to $0.49 \%{ }^{2}$

Preoperative diagnosis of uterine LMS is challenging because the disease can mimic benign LM. Differentiation of LMS and LM is required: 1) to prevent
Correspondence: Ki Hyung Kim Department of Obstetrics and Gynecology, Pusan National University School of Medicine, Busan, South Korea

Tel +82-5 I-240-7287

Fax +82-5I-248-2384

Email ghkim@pusan.ac.kr 
inadequate surgical procedures and uterine injury caused by myomectomy or power morcellation during minimally invasive hysterectomy, and 2) to prevent diagnostic delays due to misdiagnosis and facilitate prompt treatment. This differentiation is also important when choosing fertilitypreserving alternatives to hysterectomy, such as uterine artery embolization. Another important clinical issue encountered in daily practice is presumed LM with degenerative changes suspicious of LMS in patients referred from primary gynecologic clinics due to unusual sonographic appearances. In such cases, accurate differential diagnosis and correct answers are essential.

However, no known preoperative diagnostic test can reliably predict uterine LMS, although various tools have been suggested. Some clinical studies have reported that age, menopausal status, clinical presentation, tumor size, and body mass index might be helpful, but these variables do not enable diagnosis with certainty. ${ }^{3,4}$ Hence, more objective, easier, simpler tests are needed. Tumor necrosis and hemorrhage are characteristic findings of LMS, and tumor necrosis has been suggested to be associated with local inflammation, ${ }^{1}$ which suggests serum markers may reflect these associated conditions. Previously, our group reported that systemic inflammatory markers might serve as prognostic factors in ovarian and endometrial cancer and that cervical intraepithelial neoplasia has a diagnostic role in epithelial ovarian cancer (EOC). ${ }^{5-9}$ Other researchers have also reported that systemic inflammatory markers have prognostic and diagnostic values in various malignancies. ${ }^{10,11}$ However, the values of preoperative hematological parameters for the differentiation of LMS and LM have not been fully determined.

Thus, the aim of this study was to investigate the diagnostic values of preoperative clinical characteristics and hematological parameters in patients with uterine LMS.

\section{Materials and Methods Subjects}

We retrospectively reviewed the clinical records of 336 patients who had underwent surgical resection for a uterine tumor at the gynecologic oncologic centers of four participating institutions between July 2005 and August 2019. Patients with either uterine LM or LMS were eligible for inclusion in the study. Patients with uterine LM or LMS were eligible for inclusion. However, patients with any gynecologic inflammatory condition (eg, adenomyosis or endometriosis) or non-gynecologic comorbid diseases (eg, liver or heart diseases, or other malignant tumors) were excluded because of possible influences on laboratory test results. Clinical and preoperative blood test variables were subjected to analysis. All microscope slides were reviewed by an experienced gynecologic pathologist (KU Choi) to ensure consistency. This study was conducted in accordance with the ethical standards described in the Declaration of Helsinki and was approved by the Institutional Review Board (IRB) of Pusan National University Hospital and written informed consent was waived because of the retrospective nature of this study. Data were anonymized and maintained with confidentiality.

\section{Data Extraction}

Patient ages, body weights, body mass index (BMI), overweight $\left(\geq 23 \mathrm{~kg} / \mathrm{m}^{2}\right)$, and tumor size at the time of surgery were retrieved from medical records. The study population was stratified into normal body weight (BMI: 18.5$22.9 \mathrm{~kg} / \mathrm{m}^{2}$ ) and overweight or obese (BMI $\geq 23 \mathrm{~kg} / \mathrm{m}^{2}$ ) according to body mass index (BMI) categories suggested by the World Health Organization for the Asian population. Laboratory tests included: white blood cell (WBC), platelet, absolute neutrophil (ANC), absolute lymphocyte (ALC), and absolute monocyte (AMC) counts, hemoglobin $(\mathrm{Hb})$ concentration, mean corpuscular volume (MCV), red cell distribution width (RDW), mean platelet volume (MPV), platelet distribution width (PDW), C-reactive protein (CRP), lactate dehydrogenase (LDH), and cancer antigen 125 (CA125).

Laboratory tests were performed within 1 to 2 weeks prior to surgery. Quality control criteria and reference ranges adopted at the different institutions were taken into consideration while collecting laboratory results. Variables such as NLR, platelet-to-lymphocyte ratio (PLR), lymphocyte-to-monocyte ratio (LMR), and monocyte-to-lymphocyte ratio (MLR) were calculated by dividing ANC by ALC, platelet count by ALC, ALC by AMC, and AMC by ALC, respectively. ROC curve analysis was used to obtain optimal cut-off values for each variable and their derived indices for the differentiation of uterine LMS and LM.

\section{Statistical Analysis}

The Mann-Whitney- $U$ test was used to compare median values of the LMS and LM groups, and the Kruskal- 
Table I Clinical Features and Laboratory Values Between Uterine Leiomyosarcoma and Leiomyoma

\begin{tabular}{|c|c|c|c|}
\hline Pre - \& Postmenopause & LMS, Median (IQR) (n=79) & LM, Median (IQR) $(n=257)$ & $P$-value \\
\hline Age (years) & 54 & 44 & $<0.001$ \\
\hline Postmenopause (\%) & $46(58.2)$ & $4 \mid(16.0)$ & $<0.001$ \\
\hline BMI $\left(\mathrm{kg} / \mathrm{m}^{2}\right)$ & 24.65 & 23.72 & 0.145 \\
\hline Overweight $\left(\geq 23 \mathrm{~kg} / \mathrm{m}^{2}\right)$ & 49 (62.0\%) & 142 (55.5\%) & 0.369 \\
\hline WBC (per $\mu \mathrm{L})$ & 6690.0 & 5805.0 & $<0.001$ \\
\hline ANC (per $\mu \mathrm{L})$ & 4314.1 & 3387.6 & $<0.001$ \\
\hline CRP (mg/dL) & 1.00 & 0.04 & $<0.001$ \\
\hline LDH (U/L) & 425.0 & 185.5 & $<0.001$ \\
\hline NLR & 2.36 & 1.91 & $<0.001$ \\
\hline Premenopause & LMS, Median (IQR) (n=33) & LM, Median (IQR) $(n=2 \mid 6)$ & $P$-value \\
\hline BMI $\left(\mathrm{kg} / \mathrm{m}^{2}\right)$ & 23.98 & 23.53 & 0.438 \\
\hline Overweight $\left(\geq 23 \mathrm{~kg} / \mathrm{m}^{2}\right)$ & $20(60.6)$ & II $6(54.0)$ & 0.598 \\
\hline WBC (per $\mu \mathrm{L})$ & 6230.0 & 5770.0 & 0.141 \\
\hline ANC (per $\mu \mathrm{L})$ & 3970.7 & 3388.9 & 0.092 \\
\hline CRP (mg/dL) & 0.69 & 0.04 & 0.005 \\
\hline LDH (U/L) & 387.5 & 175.0 & $<0.001$ \\
\hline NLR & 2.30 & 1.95 & 0.089 \\
\hline
\end{tabular}

Note: P-values for comparisons of medians were obtained using the Mann-Whitney-U test.

Abbreviations: LMS, uterine leiomyosarcoma; LM, uterine leiomyoma; IQR, interquartile range; WBC, white blood cell; ANC, absolute neutrophil count; CRP, C-reactive protein; LDH, lactate dehydrogenase; NLR, neutrophil-lymphocyte ratio.

Wallis test was used to compare median values of three groups. The initial set of variables included in the logistic regression analysis were as follows: age at diagnosis, WBC count, $\mathrm{Hb}$ concentration, platelet count, CA125 level, NLR, PLR, LMR, and MLR. Variables such as age at diagnosis, WBC count, $\mathrm{Hb}$ concentration, platelet count, and CA125 level were dichotomized using predefined cut-off values. However, the optimal cut-off points of NLR, PLR, LMR, and MLR for predicting the presence of LMS were determined by receiver operating characteristic (ROC) curve analysis. Logistic regression analysis was used to identify variables predictive of the presence of LMS. Multivariate analysis was conducted using variables that reached significance in the univariate analysis and used to calculate odds ratios (ORs) and 95\% confidence intervals (CIs). The analysis was performed using $\mathrm{R}$ (ver. 3.6.2) software and statistical significance was accepted for $\mathrm{P}$ values of $<0.05$ throughout.

\section{Results}

Seventy-nine patients had uterine LMS and 257 patients LM. All cases were confirmed pathologically using surgical specimens. Clinical features and laboratory values for these two groups are listed in Tables 1 and 2. LMS patients were matched to LM patients who underwent hysterectomy or myomectomy during the same period. Patients with LM were also divided into LM with degeneration (92 cases) and LM without degeneration (165 cases) subgroups for comparative analysis (Table 2) because differentiation of uterine LMS from degenerated leiomyoma is an important clinical issue.

Significant differences were observed between the LMS and LM groups for the following variables: age at diagnosis, menopausal status, WBC count, ANC, CRP, LDH, and NLR (all $P<0.001$ ) (Table 1). Median patient ages in the LMS and LM groups were 54 (range, 45-61 years) and 44 years (3949 years), respectively, and the proportions of postmenopausal women were $58.2 \%$ and $16.0 \%$, respectively. In the LMS group, median WBC count, ANC, CRP, LDH, and NLR were 
Table 2 Clinical Features and Laboratory Values Between Uterine Leiomyosarcoma, Leiomyoma with Degeneration, and Leiomyoma Without Degeneration

\begin{tabular}{|c|c|c|c|c|}
\hline & \multirow[t]{2}{*}{ LMS, Median (IQR) (n=79) } & \multicolumn{2}{|c|}{ LM, Median (IQR) } & \multirow[t]{2}{*}{$P$-value } \\
\hline & & With Degeneration $(n=92)$ & Without Degeneration $(n=165)$ & \\
\hline Age (years) & $54^{\mathrm{a}}$ & $44^{\mathrm{b}}$ & $44^{\mathrm{b}}$ & $<0.001$ \\
\hline Postmenopause (\%) & $46(58.2)^{a}$ & $9(9.9)^{b}$ & $32(19.4)^{b}$ & $<0.001$ \\
\hline BMI $\left(\mathrm{kg} / \mathrm{m}^{2}\right)$ & 24.65 & 22.85 & 23.96 & 0.055 \\
\hline Overweight $\left(\geq 23 \mathrm{~kg} / \mathrm{m}^{2}\right)$ & $49(62.0 \%)$ & 44 (48.4\%) & 98 (59.4\%) & 0.144 \\
\hline WBC (per $\mu \mathrm{L})$ & $6690.0^{\mathrm{a}}$ & $6190.0^{\mathrm{a}}$ & $5620.0^{\mathrm{b}}$ & $<0.001$ \\
\hline ANC (per $\mu \mathrm{L})$ & $43 \mid 4.1^{a}$ & $3701.6^{\mathrm{b}}$ & $3283.5^{b}$ & $<0.001$ \\
\hline CRP (mg/dL) & $1.00^{\mathrm{a}}$ & $0.04^{b}$ & $0.04^{b}$ & $<0.001$ \\
\hline LDH (U/L) & $425.0^{\mathrm{a}}$ & $196.5^{\mathrm{b}}$ & $166.0^{\mathrm{b}}$ & $<0.001$ \\
\hline NLR & $2.36^{\mathrm{a}}$ & $2.00^{\mathrm{b}}$ & $1.90^{\mathrm{b}}$ & $<0.001$ \\
\hline
\end{tabular}

Notes: ${ }^{a, b}$ Medians with the same letter (superscript a, b) are not significantly different; P-values for comparisons of medians were obtained using the Kruskal-Wallis test; A post-hoc test (Bonferroni) was applied with pairwise comparison between medians.

Abbreviations: LMS, uterine leiomyosarcoma; LM, uterine leiomyoma; IQR, interquartile range; WBC, white blood cell; ANC, absolute neutrophil count; CRP, C-reactive protein; LDH, lactate dehydrogenase; NLR, neutrophil-lymphocyte ratio.

$6690 / \mu \mathrm{L} \quad(5740-8280 / \mu \mathrm{L}), \quad 4314 \mu \mathrm{L} \quad(3495-5438 / \mu \mathrm{L})$, $1.00 \mathrm{mg} / \mathrm{dL}(0.28-2.46 \mathrm{mg} / \mathrm{dL}), 425.0 \mathrm{IU} / \mathrm{L}(248.0-628.5$ IU/L), and 2.36 (1.84-3.84), respectively, and corresponding values in the LM group were $5805 / \mu \mathrm{L}(4790-7282 / \mu \mathrm{L})$, $3387 / \mu \mathrm{L}(2663-4471 / \mu \mathrm{L}), 0.04 \mathrm{mg} / \mathrm{dL}(0.02-0.12 \mathrm{mg} / \mathrm{dL})$, 185.5 IU/L (161-265 IU/L), and 1.91 (1.41-2.67), respectively. Significant differences were not observed between the LMS and LM groups for the following variables: body weight, BMI, overweight status $\left(\geq 23 \mathrm{~kg} / \mathrm{m}^{2}\right)$, and tumor size (Table 1, Supplementary Table 1). We further analyzed our data especially in premenopausal women, and our dataset appeared to be heavily unbalanced: 216 cases of LM versus 33 cases of LMS were reported. CRP and LDH showed significant differences between the LMS and LM groups (Table 1).

Significant differences were observed between the above-mentioned variables in the LMS group and the LM with or without degeneration subgroups (all $P<$ 0.001) (Table 2). After applying the Bonferroni correction, no significant differences were observed between these three groups in terms of age at diagnosis, menopausal status, ANC, CRP, LDH, or NLR (except for WBC count). However, significant differences between LMS and LM with or without degeneration were noted in terms of age at diagnosis, menopausal status, WBC count, ANC, CRP, LDH, and NLR.
Optimal thresholds, as determined by receiver operating characteristic (ROC) curve analysis, for age at diagnosis, WBC count, ANC, CRP, LDH, and NLR were; 49.5, 5940, 3404, 0.195, 339.5, and 2.157, respectively (Table 3). AUC, sensitivity, specificity, and $P$-value of each variable were shown (Table 4, Table 5). Optimal cut-off points as determined by ROC curve analyses for PLR, LMR, and MLR were; 193.6 (AUC=0.521; sensitivity 0.427, specificity $0.695), 3.344$ ( $\mathrm{AUC}=0.532$; sensitivity 0.338 , specificity 0.801 ), and 0.299 (AUC $=0.532$; sensitivity 0.338 , specificity 0.801), respectively (Figure 1, Supplementary Figure 1).

The following variables were found to be significantly associated with LMS by univariate logistic regression analyses: age at diagnosis, menopausal status, WBC count, ANC, CRP, LDH, and NLR (all $P<0.001$ ). Older or postmenopausal patients with a high WBC count, high ANC, high serum CRP, high serum LDH, and high NLR were more likely to be diagnosed with LMS. Multivariate logistic regression analyses showed postmenopausal status $(\mathrm{OR}=3.40,95 \%$ confidence interval $[\mathrm{CI}]=1.54-7.49$, $\mathrm{P}=0.002)$, high $\mathrm{WBC}$ count $(\mathrm{OR}=2.09,95 \% \mathrm{CI}=1.18-$ 3.73, $\mathrm{P}=0.012)$, high $\mathrm{ANC}(\mathrm{OR}=3.17,95 \% \mathrm{CI}=1.68-$ 6.02, $\mathrm{P}<0.001)$, high $\mathrm{CRP}(\mathrm{OR}=21.74,95 \% \mathrm{CI}=5.59-$ 84.62, $\mathrm{P}<0.001)$, high $\mathrm{LDH}(\mathrm{OR}=10.77$, 95\% $\mathrm{CI}=4.50$ 25.76, $\mathrm{P}<0.001)$, and high NLR $(\mathrm{OR}=2.58, \quad 95 \%$ $\mathrm{CI}=1.44-4.60, \mathrm{P}=0.001)$ independently predicted the presence of LMS (Table 3). 
Table 3 Univariate and Multivariate Analyses for the Discrimination of Variables That Predict Uterine Leiomyosarcoma

\begin{tabular}{|l|l|l|l|l|}
\hline \multirow{2}{*}{} & \multicolumn{1}{|c|}{ Univariate } & \multicolumn{1}{c|}{ Multivariate } \\
\cline { 2 - 5 } & \multicolumn{1}{|c|}{ OR $(95 \%$ Cl) } & \multicolumn{1}{|c|}{ P value } & OR (95\% CI) & P value \\
\hline Age (years) $(<50$ vs $\geq 50)$ & $5.46[3.18,9.38]$ & $<0.001$ & $3.40[1.54,7.49]$ & 0.002 \\
\hline Menopause $(\%)($ post vs pre) & $7.31[4.18,12.77]$ & $<0.001$ & $2.09[1.18,3.73]$ & 0.012 \\
\hline WBC (per $\mu \mathrm{L})(\leq 5940$ vs $>5940)$ & $2.72[1.59,4.67]$ & $<0.001$ & $3.17[1.68,6.02]$ & $<0.001$ \\
\hline ANC (per $\mu \mathrm{L})(\leq 3404$ vs $>3404)$ & $3.74[2.04,6.85]$ & $<0.001$ & $21.74[5.59,84.62]$ & $<0.001$ \\
\hline CRP $(\mathrm{mg} / \mathrm{dL})(\leq 0.195$ vs $>0.195)$ & $29.50[8.05,108.10]$ & $<0.001$ & $10.77[4.50,25.76]$ & $<0.001$ \\
\hline LDH $(\mathrm{U} / \mathrm{L})(\leq 339.5$ vs $>339.5)$ & $10.27[4.45,23.70]$ & $<0.001$ & $2.58[1.44,4.60]$ & 0.001 \\
\hline NLR $(\leq 2.157$ vs $>2.157)$ & $2.81[1.64,4.80]$ & $<0.001$ & \\
\hline
\end{tabular}

Note: Results of multiple logistic regression with variables show a P-value less than 0.05 in univariate regression; Multivariate model is adjusted for age.

Abbreviations: OR, odds ratio; $\mathrm{Cl}$, confidence interval; WBC, white blood cell; ANC, absolute neutrophil count; CRP, C-reactive protein; LDH, lactate dehydrogenase; NLR, neutrophil-lymphocyte ratio.

Table 4 Predictive Efficacy of Clinical Characteristics and Laboratory Data

\begin{tabular}{|l|l|l|l|l|l|l|l|l|l|}
\hline & AUC & $\mathbf{9 5 \%}$ Cl & Sensitivity & Specificity & LR+ & LR- & PPV & NPV & P-value \\
\hline Age (years) & 0.756 & $0.694-0.817$ & 0.646 & 0.750 & 2.584 & 0.137 & 0.443 & 0.873 & $<0.001$ \\
\hline WBC (per $\mu$ L) & 0.652 & $0.583-0.720$ & 0.696 & 0.543 & 1.523 & 0.200 & 0.320 & 0.853 & $<0.001$ \\
\hline ANC & 0.669 & $0.601-0.737$ & 0.784 & 0.508 & 1.593 & 0.136 & 0.315 & 0.890 & $<0.001$ \\
\hline CRP & 0.878 & $0.818-0.939$ & 0.857 & 0.831 & 5.071 & 0.028 & 0.429 & 0.975 & $<0.001$ \\
\hline LDH & 0.846 & $0.746-0.945$ & 0.667 & 0.900 & 6.670 & 0.050 & 0.818 & 0.800 & $<0.001$ \\
\hline NLR & 0.645 & $0.575-0.715$ & 0.635 & 0.617 & 1.658 & 0.220 & 0.324 & 0.854 & $<0.001$ \\
\hline NLR+LDH & 0.863 & $0.746-0.945$ & 0.667 & 0.900 & 6.670 & 0.050 & 0.818 & 0.800 & 0.049 \\
\hline NLR+LDH+Age & 0.864 & $0.761-0.955$ & 0.926 & 0.700 & 3.087 & 0.024 & 0.676 & 0.933 & 0.049 \\
\hline
\end{tabular}

Abbreviations: AUC, area under curve; Cl, confidence interval; WBC, white blood cell; ANC, absolute neutrophil count; CRP, C-reactive protein; LDH, lactate dehydrogenase; NLR, neutrophil-lymphocyte ratio; LR+, Positive likelihood ratio; LR-, Negative likelihood ratio; PPV, positive predictive value; NPV, negative predictive value.

Among the values for each combination markers, NLR $+\mathrm{LDH}$ and $\mathrm{NLR}+\mathrm{LDH}+$ Age were found to have $\mathrm{AUC}=0.845$, sensitivity 0.667 , specificity 0.900 and $\mathrm{AUC}=0.858$, sensitivity 0.926 , specificity 0.700 , respectively (Table 4 ).

\section{Discussion}

Although previous studies have reported that various tools might differentiate LMS and LM, results are debatable, and no evidence has been presented that any tool can distinguish LMS from benign LM. In this study, univariate and multivariate logistic regression analyses showed that age at diagnosis $\geq 50$ years old, postmenopausal status, $\mathrm{WBC} \geq 5940(/ \mu \mathrm{L}), \mathrm{ANC} \geq 3404(/ \mu \mathrm{L}), \mathrm{CRP} \geq 0.195$ $(\mathrm{mg} / \mathrm{dL}), \mathrm{LDH} \geq 339.5(\mathrm{U} / \mathrm{L})$, and NLR $\geq 2.157$ significantly predicted the presence of LMS.
LMS is often incidentally confirmed following initial surgery performed under the presumption of LM, and many cases of uterine LMS are often initially managed conservatively without hysterectomy based on this presumption. As has been previously recommended, physicians should provide patients with more information about uterine sarcoma risks when recommending hysterectomy for benign disease, ${ }^{12}$ as inadequate surgical procedures, especially those involving uterine injury such as myomectomy or morcellation, during minimally invasive surgery worsen prognoses. When LMSs are misdiagnosed as benign lesions, delayed diagnosis and inadequate treatment will inevitably be followed by poorer prognoses.

Given the aggressive nature of LMS, early detection strategies have been the focus of several recently 


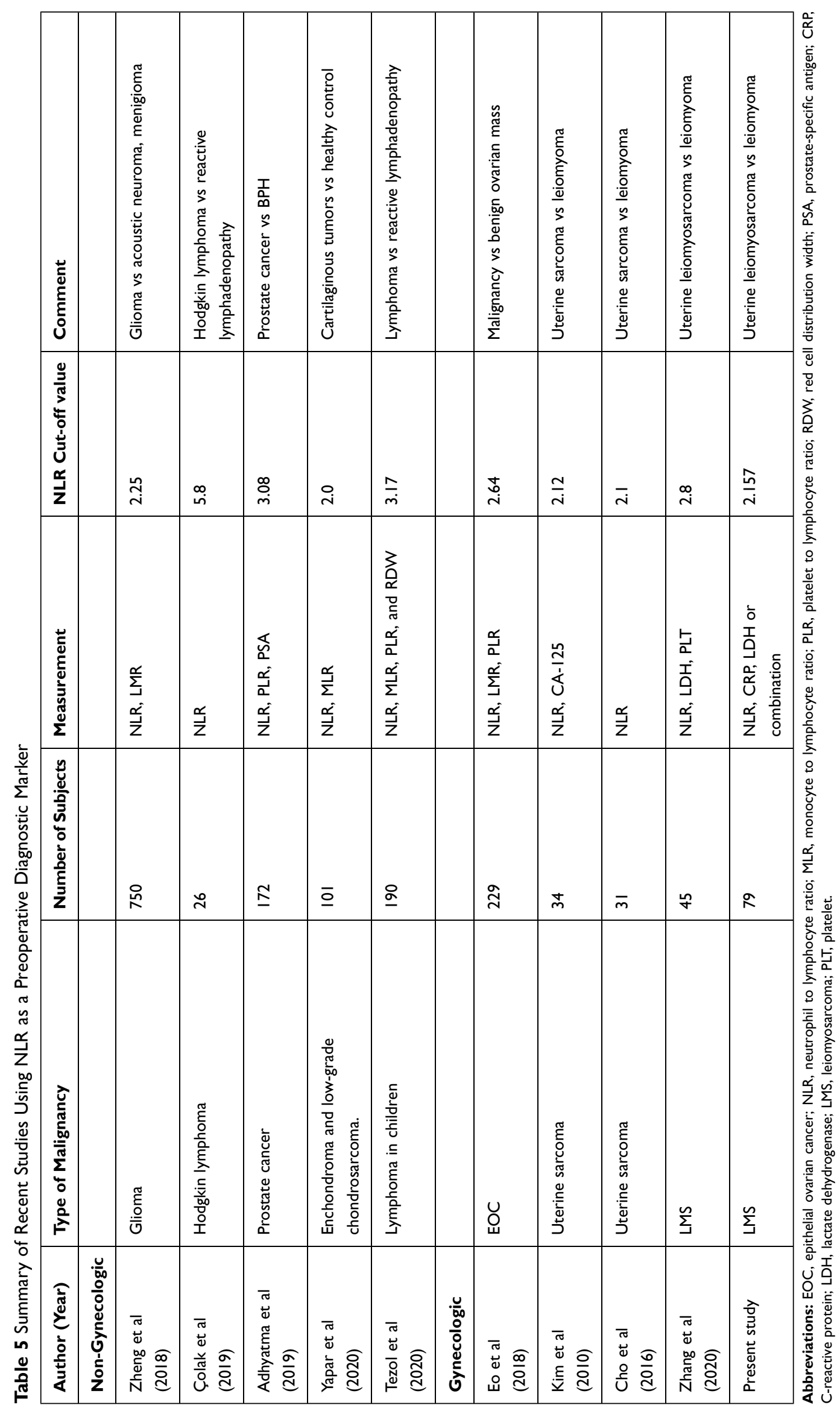


A

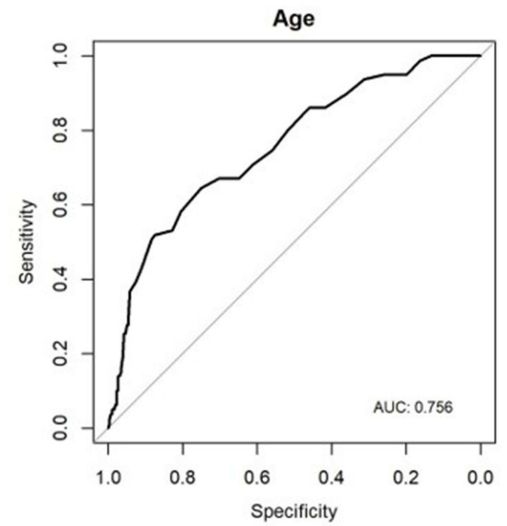

C

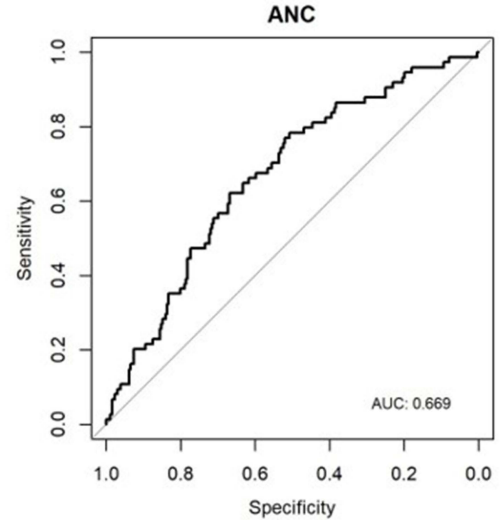

E

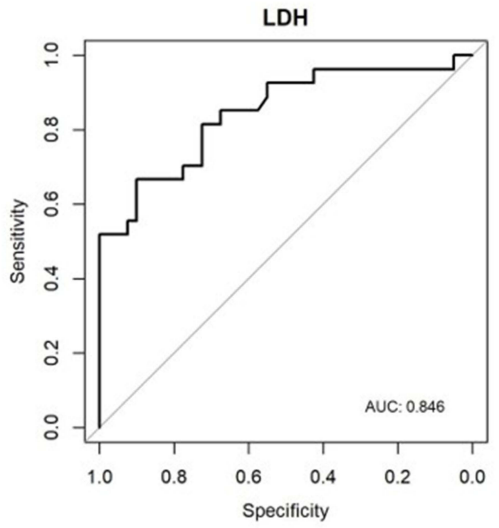

G

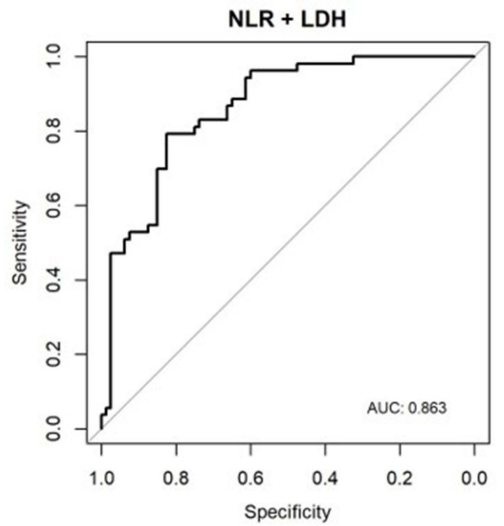

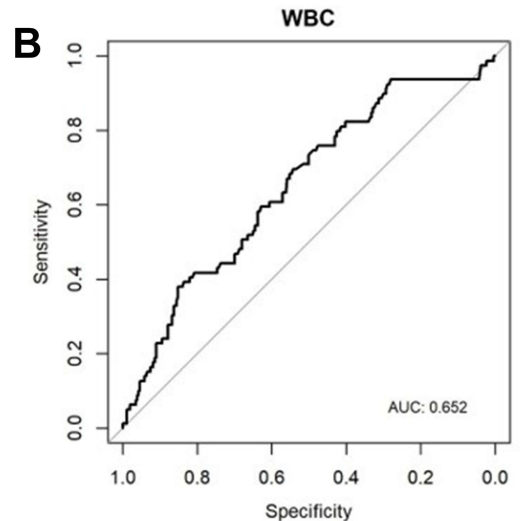

D

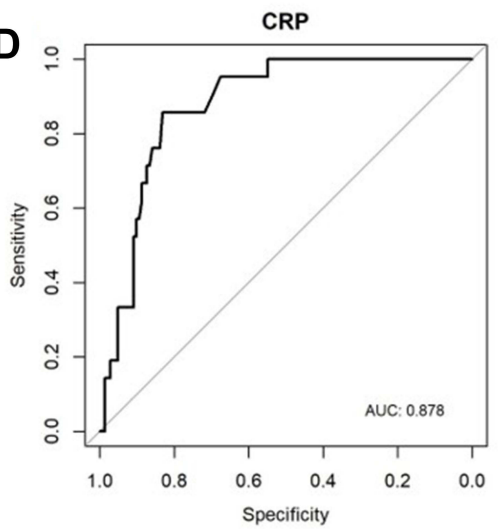

$\mathbf{F}$
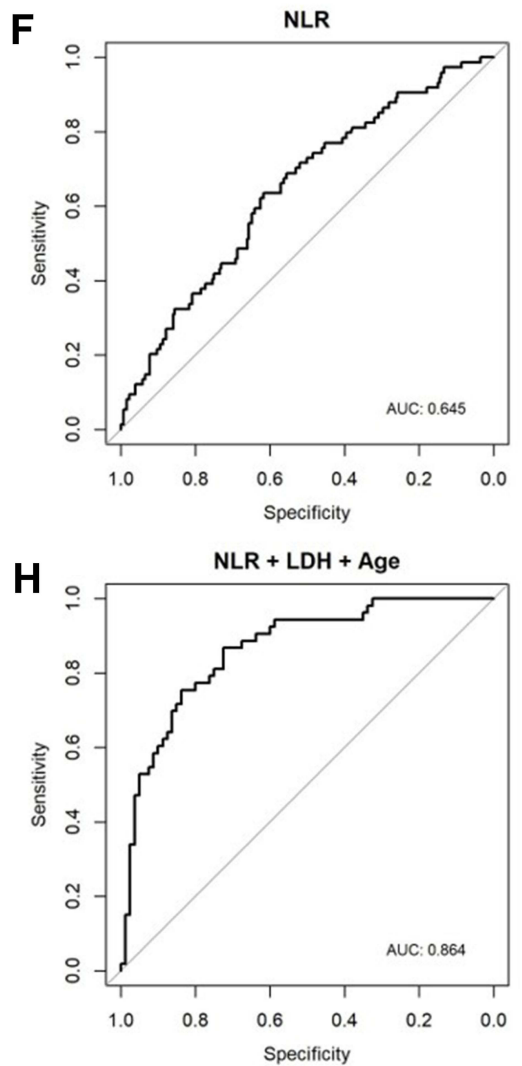

Figure I Receiver operating characteristic (ROC) curves for (A) age at diagnosis, (B) WBC count, (C) absolute neutrophil count (ANC), (D) C-reactive protein (CRP), (E) lactate dehydrogenase (LDH), (F) neutrophil-to-lymphocyte ratio (NLR), (G) LDH+NLR, and (H) LDH+NLR+Age. 
published studies. ${ }^{13}$ Currently, no reliable diagnostic tool is available for differentiating uterine LMS and LM prior to surgery. In a Norwegian cohort study, approximately $54 \%$ of uterine LMS cases were not identified before surgery, ${ }^{14}$ which adequately demonstrates a reliable preoperative tool is urgently needed to choose the correct surgical pathway and ensure oncological safety.

Clinical presentations of, for example, abnormal uterine bleeding, abdominal pain, and rapid tumor growth, encourage suspicions of LMS, but these manifestations can also occur in patients with LM. A preoperative LMS risk scoring system based on sonography findings and basic clinical characteristics has been reported, ${ }^{15}$ and the authors recommended the adoption of stepwise diagnostic procedures, such as endometrial biopsy, serum LDH, Doppler sonography, or MRI in patients at perceived risk of LMS.

The prevalence of LMS increases with age and rates of LMS at surgery for presumed LM have been reported for different age groups. In women under 40 years, between 40 and 49 years, and over 49 years old, reported prevalence of occult LMS were $0 \%, 0.15 \%$, and $1.2 \%$, respectively, ${ }^{16}$ which suggests that age may be significant. Previous studies have also reported that an age of $\geq 40$ or $\geq 45$ years independently predicted LMS, ${ }^{4,17}$ and our findings concur with these reports. In the present study, patients in LMS group were older than those in the LM group ( 54 vs 44 years old, $P<0.001$ ), and an age of $\geq 50$ years independently predicted the presence of LMS (Table 3). Similarly, menopausal status has also been reported to be associated with LMS. $^{18}$ Multinu et al showed that the rates of unexpected LMS during surgery for LM among premenopausal and peri/postmenopausal women were $0.35 \%$ and $0.57 \%$, respectively. ${ }^{17}$ In our study, the LMS group had a higher percentage of postmenopausal women $(58.2 \%$ vs $16.0 \%, \mathrm{p}<0.001)$.

Frequently, LMS cannot be distinguished from LM using clinical criteria alone. Although diverse imaging modalities, such as Doppler sonography, MRI, and positron emission tomography have been recently suggested as ancillary diagnostic aids for LMS, their preoperative predictive roles remain unclear. ${ }^{19,20}$ Furthermore, these diagnostic tools cannot be applied to all patients with a uterine tumor when differentiation is required because of their costs and limited accessibilities. No radiographic criteria have been identified that enable definitive differentiation. ${ }^{21}$ Ultrasonography is a commonly used first imaging modality in gynecology, but its diagnostic role is limited by the similar sonographic appearances of LM and LMS. ${ }^{22}$ Of these imaging modalities, recent studies have shown that some MRI features may allow the differentiation of LMS and LM. ${ }^{22}$ The characteristics of LMS on MR images include margins and shapes, signal intensities in T1 and T2weighted images, enhancement in gadolinium contrastenhanced images, and apparent diffusion coefficient (ADC) values. Nonetheless, despite current imaging advances, confident diagnosis of LMS remains challenging because the appearance of LMS in MR images is variable and the images features of degenerated leiomyoma and overlap. ${ }^{19}$ In this retrospective analysis, the diagnostic role of MRI was not assessed because it was not performed in most of the study subjects.

Studies on associations between laboratory variables and the preoperative differentiation of uterine LMS are lacking. Some indicators in peripheral blood are known to reflect inflammatory response in the tumor microenvironment, ${ }^{23}$ and CRP is one such systemic inflammatory marker. Tumor cell necrosis and subsequent inflammation in cancer tissue may be associated with CRP elevation, and elevated CRP pretreatment might be a prognostic marker in patients with soft tissue sarcoma. ${ }^{24}$ Furthermore, a small number of studies have suggested that serum CRP might be useful for the preoperative differentiation of uterine sarcomas. ${ }^{1,25}$ However, its diagnostic accuracy remains controversial. In the present study, CRP level in LMS group was significantly higher than in the LM group ( 1.00 vs $0.04 \mathrm{mg} / \mathrm{dL}, \mathrm{P}<0.001)$, and a CRP level of $\geq 0.195(\mathrm{mg} / \mathrm{dL})$ independently predicted LMS (Tables 1 and 3).

LDH has been shown to be useful for diagnosing and predicting the outcomes of patients with various malignancies. ${ }^{4}$ This enzyme is involved in the metabolism of cancer cells and causes these cells to suppress and evade the immune system by altering tumor microenvironments. ${ }^{26}$ Although studies have reported significant differences between LDH levels in LM and LMS patients, ${ }^{4,15,21}$ the cutoff values used varied and no LDH value has been defined for LMS. In these studies, LDH levels of level $\geq 193$ U/L, > 279 $\mathrm{U} / \mathrm{L}$, and $\geq 300 \mathrm{U} / \mathrm{L}$ were reported to independently predict the presence of uterine sarcoma preoperatively. In the present study, mean LDH level in the LMS group was higher than in the LM group ( 425.0 vs $185.5 \mathrm{U} / \mathrm{L}, P<0.001$ ) and a level of $\geq$ $339.5 \mathrm{U} / \mathrm{L}$ independently predicted the presence of LMS (Tables 1 and 3). Recent studies further assessed the role of LDH isoenzymes in discriminating between benign uterine masses and sarcomas. ${ }^{27,28} \mathrm{LDH}$ isoenzyme levels, especially LDH3, significantly differed between both conditions and elevated LDH5/LDH1 ratio was indicative of sarcoma. 
These studies suggested that LDH isoenzymes assessment may be relevant in preoperative diagnosis of uterine sarcoma.

It is now widely accepted that inflammation and cancer are closely associated, and increasing evidence indicates chronic inflammation plays a crucial role in carcinogenesis, tumor progression, therapeutic response, and clinical outcome, ${ }^{5,6}$ which suggests markers of systemic inflammation might provide useful information regarding the presence of malignancy. Tumor necrosis and hemorrhage are characteristics of LMS, and the former is associated with local inflammation, ${ }^{1}$ which suggests serum markers may reflect these conditions. Furthermore, some hematological changes, such as reduced lymphocyte count and increased neutrophil count, are observed in patients with malignancies, and it has been established that neutrophils participate in the link between inflammation and cancer and the creation of a tumor microenvironment that favors angiogenesis, cancer progression, and metastasis..$^{29}$ On the other hand, lymphocytes are involved in cell-mediated response to tumor infiltration, and a low lymphocyte count might result in inadequate immune response, and thus, be associated with unfavorable outcomes. ${ }^{11}$ In the present study, lymphocyte counts were similar in LMS and LM, but WBC and neutrophil counts were higher in LMS patients (Tables 1 and 3), which is in accord with previous studies that reported an elevated neutrophil count was associated with a preoperative diagnosis of LMS., ${ }^{4,18,30}$ The cutoffs of WBC and ANC in this study are in the normal range. Systemic inflammatory markers in this study may reflect underlying inflammatory condition, but do not necessarily mean current or present overt inflammatory status. Similar cutoff values of WBC and ANC in the normal range were observed in previous studies. ${ }^{4,31} \mathrm{WBC}$ count $\geq 5700 / \mu \mathrm{L}$ was significant predictive factor for sarcoma and neutrophil count $(5400 \pm 3200$ vs $3500 \pm 1200 / \mu \mathrm{L}, P<0.001)$ in LMS group was significantly higher than control group.

Recent studies indicate that NLR might be a useful diagnostic and prognostic marker for different cancers. ${ }^{11,32-36}$ A high NLR represents an increase in immunosuppressive status, and reportedly, is associated with poor survival in esophageal, gastric, colorectal, lung, breast cancer, and urologic cancers. ${ }^{32,34}$ Regarding gynecologic malignancies, high NLR is associated with poor clinical outcomes in ovarian, endometrial, and cervical cancer, and in uterine sarcoma. ${ }^{11,32,33,35}$ In a metaanalysis conducted by $\mathrm{Wu}$ et al in cervical cancer, an elevated NLR was found to be closely associated with poor clinical outcomes and unfavorable clinicopathological factors. ${ }^{36}$ Similarly, Jeong et al reported that an elevated preoperative NLR $(\geq 2.60)$ was associated with poor clinical outcomes in uterine sarcoma patients. ${ }^{11}$

However, few studies have investigated the diagnostic values of markers of systemic inflammation in different cancers (Table 5). In non-gynecologic cancers, NLR has been suggested to predict the presence of Hodgkin lymphoma, glioma, prostate cancer, or cartilaginous tumors (enchondroma and low-grade chondrosarcoma), ${ }^{10,37-40}$ whereas in gynecologic cancers, we have reported on the usefulness of NLR as a diagnostic marker for the presence of EOC. ${ }^{8}$ Kim et al described the efficacy of NLR for the preoperative diagnosis of uterine sarcomas and suggested that NLR $(\geq 2.12)$ might provide a more useful and costeffective means than serum CA-125 for their preoperative differentiation. ${ }^{30}$ Cho et al concluded that an NLR of $>2.1$ significantly and independently predicted the presence of uterine sarcoma, ${ }^{3}$ and interestingly, Zhang et al reported that an NLR of $\geq 2.8$ independently predicted LMS. ${ }^{4}$ Similarly, we found that an NLR of $\geq 2.157$ might be useful for differentiating LMS and LM. The reported NLR cut-off values for NLR are similar to those obtained in the present study, whereas the cut-off value reported by Zhang et al was slightly higher (Table 5).

Uterine LMS is challenging to diagnose preoperatively and can mimic the appearance of benign uterine LM clinically and radiologically. Recently, Fujibuchi et al reported elevated WBC count, serum CRP level, and serum LDH level were independent predictive markers of soft tissue sarcomas and recommended that patients with three or more predictive factors should be referred to a specialist. ${ }^{31}$ In the present study, multivariate analysis revealed preoperative CRP $>0.195, \mathrm{LDH}>339.5$, or NLR $>2.195$ independently predicted the presence of LMS (Table 3), and that other clinical and laboratory variables, including lymphocyte count, monocyte count, tumor size, platelet count, $\mathrm{Hb}$ concentration, $\mathrm{RDW}$, and CA125 level did not (Supplementary Tables S1 and S2). However, it should be noted that the reported significances of $\mathrm{Hb}$ concentration and tumor size vary between studies. $^{3,4,18,31}$ LMR, MLR, and PLR were analyzed in this study, but these did not show significant differences.

Recently, studies focused on the prognostic biomarkers or models have been reported in uterine sarcoma because clinical characteristics of sarcoma patients could not effectively distinguish between patients with high or low survival rates, and effective and responsible prognostic biomarkers are few. Zhou et al identified an RNA-Seq expression signature for uterine sarcoma to improve 
clinical outcomes. ${ }^{41}$ Furthermore, multiple somatic mutations and copy-number alterations that are therapeutic targets for uterine carcinosarcoma were identified. ${ }^{42}$

One strength of the present study is that we divided the LM group into LM with or without degeneration subgroups and investigated differences between clinical and laboratory variables in the LMS and LM with degeneration groups because this differentiation is an important clinical issue and no previous study has addressed the topic. However, our study also has several limitations that warrant consideration. First, we were unable to confirm that preoperative WBC, ANC, CRP, LDH, and NLR differentiate LMS from LM because the data used were collected retrospectively. Second, these serum markers are non-specific markers of inflammation, and although patients with any inflammatory conditions, such as adenomyosis or endometriosis, were excluded, laboratory test results may have been affected by other unrecognized systemic inflammatory conditions. Third, due to the rarity of this disease, the sample sizes in this study were relatively small and as a result we were unable to accurately determine CRP, $\mathrm{LDH}$, or NLR cut-off values in the presence of LMS. Accordingly, the cut-off values obtained by ROC curve analysis may differ from those reported previously.

In summary, our findings suggest that in older or postmenopausal patients, high WBC count, ANC, CRP, LDH, and NLR levels should be considered independent biomarkers for the differentiation of LMS and LM. Considering that these serum markers are readily measured and calculated, inexpensive, and universally available in clinical settings, they may be of considerable value, although the topic needs further investigation. Easily accessible tools for differentiating LMS and LM are important for primary care physicians that care for women with a uterine tumor. Successful prediction of the presence of uterine LMS using these simple clinical and laboratory markers might prevent misdiagnoses and improve prognoses.

\section{Acknowledgments}

This work was supported by clinical research grant from Pusan National University Hospital in 2021.

\section{Author Contributions}

All authors made a significant contribution to the work reported, whether that is in the conception, study design, execution, acquisition of data, analysis and interpretation, or in all these areas; took part in drafting, revising or critically reviewing the article; gave final approval of the version to be published; have agreed on the journal to which the article has been submitted; and agree to be accountable for all aspects of the work.

\section{Disclosure}

The authors declare that they have no competing interests.

\section{References}

1. Nishigaya Y, Kobayashi Y, Matsuzawa Y, et al. Diagnostic value of combination serum assay of lactate dehydrogenase, D-dimer, and Creactive protein for uterine leiomyosarcoma. J Obstet Gynaecol Res. 2019;45(1):189-194. doi:10.1111/jog.13792

2. Cui RR, Wright JD. Risk of occult uterine sarcoma in presumed uterine fibroids. Clin Obstet Gynecol. 2016;59(1):103-118. doi:10.1097/GRF.0000000000000163

3. Cho HY, Kim K, Kim YB, No JH. Differential diagnosis between uterine sarcoma and leiomyoma using preoperative clinical characteristics. J Obstet Gynaecol Res. 2016;42(3):313-318. doi:10.1111/jog.12915

4. Zhang G, Yu X, Zhu L, Fan Q, Shi H, Lang J. Preoperative clinical characteristics scoring system for differentiating uterine leiomyosarcoma from fibroid. BMC Cancer. 2020;20:1-7.

5. Eo WK, Chang HJ, Kwon SH, et al. The lymphocyte-monocyte ratio predicts patient survival and aggressiveness of ovarian cancer. $J$ Cancer. 2016;7(3):289. doi:10.7150/jca.13432

6. Eo WK, Kwon S, Koh SB, et al. The lymphocyte-monocyte ratio predicts patient survival and aggressiveness of endometrial cancer. $J$ Cancer. 2016;7(5):538. doi:10.7150/jca.14206

7. Chun S, Shin K, Kim KH, et al. The neutrophil-lymphocyte ratio predicts recurrence of cervical intraepithelial neoplasia. $J$ Cancer. 2017;8(12):2205. doi:10.7150/jca.19173

8. Eo WK, Kim KH, Park EJ, et al. Diagnostic accuracy of inflammatory markers for distinguishing malignant and benign ovarian masses. $J$ Cancer. 2018;9(7):1165. doi:10.7150/jca.23606

9. Kwon BS, Jeong DH, Byun JM, et al. Prognostic value of preoperative lymphocyte-monocyte ratio in patients with ovarian clear cell carcinoma. J Cancer. 2018;9(7):1127. doi:10.7150/jca.24057

10. Yapar A, Ulucaköy C, Sezgin EA, Atalay İB, Ekşioğlu MF. Diagnostic role of neutrophil-to-lymphocyte ratio and monocyte-tolymphocyte ratio in patients with enchondroma and low-grade chondrosarcoma. Joint Dis Relat Surg. 2020;31(2):286. doi:10.5606/ ehc. 2020.73629

11. Jeong MJ, Park JH, Hur SY, Kim CJ, Nam HS, Lee YS. Preoperative neutrophil-to-lymphocyte ratio as a prognostic factor in uterine sarcoma. J Clin Med. 2020;9(9):2898. doi:10.3390/jcm9092898

12. Gallicchio L, Harvey LA, Kjerulff KH. Fear of cancer among women undergoing hysterectomy for benign conditions. Psychosom Med. 2005;67(3):420-424. doi:10.1097/01.psy.0000160472.69303.56

13. Ricci S, Stone RL, Fader AN. Uterine leiomyosarcoma: epidemiology, contemporary treatment strategies and the impact of uterine morcellation. Gynecol Oncol. 2017;145(1):208-216. doi:10.1016/j. ygyno.2017.02.019

14. Skorstad M, Kent A, Lieng M. Uterine leiomyosarcoma-incidence, treatment, and the impact of morcellation. A nationwide cohort study. Acta Obstet Gynecol Scand. 2016;95(9):984-990. doi:10.1111/ aogs. 12930

15. Köhler G, Vollmer M, Nath N, et al. Benign uterine mass-discrimination from leiomyosarcoma by a preoperative risk score: a multicenter cohort study. Arch Gynecol Obstet. 2019;300 (6):1719-1727. doi:10.1007/s00404-019-05344-0

16. Valzacchi GMR, Rosas P, Uzal M, Gil SJ, Viglierchio VT. Incidence of leiomyosarcoma at surgery for presumed uterine myomas in different age groups. J Minim Invasive Gynecol. 2020;27(4):926-929. doi:10.1016/j.jmig.2019.06.013 
17. Multinu F, Casarin J, Tortorella L, et al. Incidence of sarcoma in patients undergoing hysterectomy for benign indications: a population-based study. Am J Obstet Gynecol. 2019;220(2):179.e1e10. doi:10.1016/j.ajog.2018.11.1086

18. Lawlor H, Ward A, Maclean A, et al. Developing a preoperative algorithm for the diagnosis of uterine leiomyosarcoma. Diagnostics. 2020;10(10):735. doi:10.3390/diagnostics10100735

19. DeMulder D, Ascher SM. Uterine leiomyosarcoma: can MRI differentiate leiomyosarcoma from benign leiomyoma before treatment? Am J Roentgenol. 2018;211(6):1405-1415. doi:10.2214/ AJR.17.19234

20. Lee JW, Park JY, Lee HJ, et al. Preoperative [18 F] FDG PET/CT tumour heterogeneity index in patients with uterine leiomyosarcoma: a multicentre retrospective study. Eur J Nucl Med Mol Imaging. 2018;45(8):1309-1316. doi:10.1007/s00259-018-3975-6

21. Nagai T, Takai Y, Akahori T, et al. Novel uterine sarcoma preoperative diagnosis score predicts the need for surgery in patients presenting with a uterine mass. Springerplus. 2014;3(1):678. doi:10.1186/ 2193-1801-3-678

22. Sun S, Bonaffini P, Nougaret S, et al. How to differentiate uterine leiomyosarcoma from leiomyoma with imaging. Diagn Interv Imaging. 2019;100(10):619-634. doi:10.1016/j.diii.2019.07.007

23. Ohtani H. Focus on TILs: prognostic significance of tumor infiltrating lymphocytes in human colorectal cancer. Cancer Immunity Arch. 2007;7(1).

24. Wang X, Liu S, Zhao X, Fang E, Zhao X. The value of C-reactive protein as an independent prognostic indicator for disease-specific survival in patients with soft tissue sarcoma: a meta-analysis. PLoS One. 2019;14(7):e0219215. doi:10.1371/journal.pone.0219215

25. Glorie N, Baert T, Van Den Bosch T, Coosemans A. Circulating protein biomarkers to differentiate uterine sarcomas from leiomyomas. Anticancer Res. 2019;39(8):3981-3989. doi:10.21873/ anticanres. 13553

26. Ding J, Karp JE, Emadi A. Elevated lactate dehydrogenase (LDH) can be a marker of immune suppression in cancer: interplay between hematologic and solid neoplastic clones and their microenvironments. Cancer Biomark. 2017;19(4):353-363. doi:10.3233/CBM-160336

27. Di Cello A, Borelli M, Marra ML, et al. A more accurate method to interpret lactate dehydrogenase $(\mathrm{LDH})$ isoenzymes' results in patients with uterine masses. Eur J Obstet Gynecol Reprod Biol. 2019;236:143-147. doi:10.1016/j.ejogrb.2019.03.017

28. Mollo A, Raffone A, Travaglino A, et al. Increased LDH5/LDH1 ratio in preoperative diagnosis of uterine sarcoma with inconclusive MRI and LDH total activity but suggestive CT scan: a case report. BMC Womens Health. 2018;18(1):169. doi:10.1186/s12905-0180662-5

29. El-Kenawi A, Hänggi K, Ruffell B. The immune microenvironment and cancer metastasis. Cold Spring Harb Perspect Med. 2020;10(4): a037424. doi:10.1101/cshperspect.a037424

30. Kim HS, Han K, Chung HH, et al. Neutrophil to lymphocyte ratio for preoperative diagnosis of uterine sarcomas: a case-matched comparison. Eur J Surg Oncol. 2010;36(7):691-698. doi:10.1016/j. ejso.2010.05.004
31. Fujibuchi T, Miyawaki J, Kidani T, Imai H, Miura H. Prediction of soft tissue sarcoma from clinical characteristics and laboratory data. Cancers. 2020;12(3):679. doi:10.3390/cancers12030679

32. Cong R, Kong F, Ma J, Li Q, Wu Q, Ma X. Combination of preoperative neutrophil-lymphocyte ratio, platelet-lymphocyte ratio and monocyte-lymphocyte ratio: a superior prognostic factor of endometrial cancer. BMC Cancer. 2020;20:1-11. doi:10.1186/s12885020-06953-8

33. Chen W, Zhong S, Shan B, et al. Serum D-dimer, albumin and systemic inflammatory response markers in ovarian clear cell carcinoma and their prognostic implications. J Ovarian Res. 2020;13 (1):89. doi:10.1186/s13048-020-00693-w

34. Su S, Liu L, Li C, Zhang J, Li S. Prognostic role of pretreatment derived neutrophil to lymphocyte ratio in urological cancers: a systematic review and meta-analysis. Int $J$ Surg. 2019;72:146-153. doi:10.1016/j.ijsu.2019.10.043

35. Baert T, Van Camp J, Vanbrabant L, et al. Influence of CA125, platelet count and neutrophil to lymphocyte ratio on the immune system of ovarian cancer patients. Gynecol Oncol. 2018;150 (1):31-37. doi:10.1016/j.ygyno.2018.05.004

36. Wu J, Chen M, Liang C, Su W. Prognostic value of the pretreatment neutrophil-to-lymphocyte ratio in cervical cancer: a meta-analysis and systematic review. Oncotarget. 2017;8(8):13400. doi:10.18632/ oncotarget.14541

37. Tezol O, Bozlu G, Sagcan F, Tuncel DF, Citak C. Value of neutrophil-to-lymphocyte ratio, monocyte-to-lymphocyte ratio, platelet-to-lymphocyte ratio and red blood cell distribution width in distinguishing between reactive lymphadenopathy and lymphoma in children. Bratisl Lek Listy. 2020;121(4):287. doi:10.4149/ BLL_2020_045

38. Zheng SH, Huang JL, Chen M, Wang BL, Ou QS, Huang SY. Diagnostic value of preoperative inflammatory markers in patients with glioma: a multicenter cohort study. J Neurosurg. 2018;129 (3):583-592. doi:10.3171/2017.3.JNS161648

39. Çolak M, Eravcı FC, Karakurt SE, Karakuş MF, Ikincioğulları A, Özcan KM. The predictive value of neutrophil-to-lymphocyte ratio for Hodgkin's lymphoma diagnosis in patients with asymptomatic cervical lymphadenopathy. Indian J Otolaryngol Head Neck Surg. 2019;71(1):986-991. doi:10.1007/s12070-019-01676-0

40. Adhyatma KP, Prapiska FF, Siregar GP, Warli SM. Systemic inflammatory response in predicting prostate cancer: the diagnostic value of neutrophil-to-lymphocyte ratio. Open Access Maced J Med Sci. 2019;7(10):1628. doi:10.3889/oamjms.2019.177

41. Zhou JG, Zhao HT, Jin SH, Tian X, Ma H. Identification of a RNA-seq-based signature to improve prognostics for uterine sarcoma. Gynecol Oncol. 2019;155(3):499-507. doi:10.1016/j. ygyno.2019.08.033

42. Cherniack AD, Shen H, Walter V, et al. Integrated molecular characterization of uterine carcinosarcoma. Cancer Cell. 2017;31 (3):411-423. doi:10.1016/j.ccell.2017.02.010
Cancer Management and Research is an international, peer-reviewed open access journal focusing on cancer research and the optimal use of preventative and integrated treatment interventions to achieve improved outcomes, enhanced survival and quality of life for the cancer patient.
The manuscript management system is completely online and includes a very quick and fair peer-review system, which is all easy to use. Visit http://www.dovepress.com/testimonials.php to read real quotes from published authors. 